\title{
A Proposed Coil System for the Improved Realization of the Absolute Ampere
}

\author{
P. T. Olsen, * W. D. Phillips* and E. R. Williams*
}

National Bureau of Standards, Washington, D.C. 20234

April 1, 1980

\begin{abstract}
In order to resolve the discrepancies which presently exist between the directly measured values of the absolute or SI ampere and the calculated values obtained indirectly from other fundamental physical constant determinations, one must design an absolute ampere experiment which will produce a result with an uncertainty of one half part per million or less. A new approach recently proposed by Kibble promises such sub-ppm accuracy. Presented here is the design and evaluation of a coil system which will fulfill the requirements of this new approach.
\end{abstract}

Key words: Absolute ampere; current balance; magnetic force; radial magnetic field; superconducting coils.

\section{Introduction}

One of the first experiments conducted at the National Bureau of Standards (NBS) was that of determining the as maintained unit of electrical current in terms of the absolute ampere. To date three different methods have been employed: the classical current balance, the ratio-of-radii method, and the electrodynamometer method.

\subsection{The classical current balance}

A pair of equal diameter coaxial coils with their axes vertical and electrically connected so that their axial magnetic fields are in opposition, produce a purely radial magnetic field at the mid-plane. A third coil (also with its axis vertical) is suspended from the arm of a precision balance in this radial magnetic field. The interaction between the magnetic field produced by the coil pair and the current in the suspended coil produces a vertical force on the suspended coil that is measured with the balance. The expected force is calculated from the measured physical dimensions of the coil system. The difference in the actual force from that calculated is an indication of the difference between the laboratory, or as-maintained unit of current, and the absolute ampere as defined in the Systeme International d'Unites (SI) [1]. ${ }^{1}$

\footnotetext{
- Center for Absolute Physical Quantities National Measurement Laboratory.

${ }^{1}$ Figures in brackets indicate literature references at the end of this paper.
}

\subsection{The ratio-of-radii method}

The ratio-of-radii technique is in general identical to the classical current balance except that the coils do not have to be measured quite so carefully. Only the ratio of the radii of the coils producing the radial field to that of the suspended coil needs to be measured. This ratio is determined by measuring the ratio of currents in the field and suspended coils needed to produce a null field at the center. The critical dimensional measurement here is of the centrally located probe used as the zero field sensor since the finite size of this object significantly influences the accuracy of the ratio [2].

\subsection{The electrodynamometer method}

The Pellat balance or electrodynamometer consists of a small coil mounted on a balance beam. The coil axis is positioned orthogonally to a uniform magnetic field at the center of a precision solenoid. In this technique, the torque on the small coil is measured. The torque constant of the balance is calculated from the dimensional measurements of the coils and balance beam $[3,4]$.

Common to all three methods is the small mass used as a restoring force on the scale pan of the balance, typically one to two grams. The mass of the system on the balance beam is approximately one kilogram, so a weighing of 1 part in $10^{9}$ is necessary in order to just achieve an experimental uncertainty of 1 part in $10^{6}$ or $1 \mathrm{ppm}$ (part per million). Since a 
weighing of 1 part in $10^{9}$ is not readily achievable, the previous experiments were already limited to several ppm uncertainty. This restriction, combined with the tedious measurements for determining the actual size of some physical object, limited the accuracy of all previous experiments to a value considerably in excess of $1 \mathrm{ppm}$.

In addition to making direct current balance measurements of $K_{A}$, the ratio of the as-maintained ampere to the absolute or SI ampere, it is possible to compute a value of $K_{A}$ through the route of other measured fundamental constants. At the present time, these computed values of $K_{A}$ exhibit much less uncertainty than the several direct measurements of $K_{A}$. Yet these derived values of $K_{A}$ generally disagree among themselves and they in turn disagree with the direct current balance determinations of $K_{A}$ [5].

In light of the recent advances in technological tools such as the computer, laser interferometry, and cryogenic techniques, it appears that a conventional current balance experiment could be conducted to yield a result for $K_{A}$ with an uncertainty of better than $1 \mathrm{ppm}$. Examinations of past experiments indicates that the chances of success would greatly improve if: (a) the electromagnetic force to be measured was increased by two or three orders of magnitude; and (b) the need for super-accurate dimensional measurements of a physical body of inconvenient geometrical shape were lessened or eliminated.

B. P. Kibble of the National Physical Laboratory (NPL), has proposed a new method of conducting an absolute ampere experiment which appears to have the capability of measuring $K_{A}$ to an uncertainty of significantly less than 1.0 ppm [6,7]. Kibble's approach eliminates the need to measure the physical size of coils and allows a three order of magnitude increase in the electromagnetic force. This paper follows Kibble's general approach and presents a unique coil geometry by which the absolute ampere should be measurable to an accuracy approaching $0.1 \mathrm{ppm}$.

\section{Theory}

Although the new method proposed by Kibble has been published, for the sake of clarity the idea is briefly developed here.

A length of wire, $\ell$, traversing a uniform magnetic field, $\mathbf{B}$, at a constant velocity, $\mathbf{v}$, has a voltage potential difference $\varepsilon$ induced at its ends according to

$$
\varepsilon=\ell \cdot(\mathbf{B} \times \mathbf{v})=\mathbf{v} \cdot(\boldsymbol{l} \times \mathbf{B}) .
$$

When the wire is stationary and a steady current, $I$, flows within it, a force is exerted on the wire given by

$$
\mathbf{F}=I \ell \times \mathbf{B}
$$

It is sufficient for our purposes to examine the situation where $\ell, \mathbf{B}$, and $\mathbf{v}$ are orthogonal. Both $\ell$ and $\mathbf{B}$ are the same in eqs (1) and (2), so eq (2) can be written as

$$
F=\varepsilon I / v \text {. }
$$

Applying the above equations to the classical current balance, one can imagine a coil of $N$ turns and radius $a_{o}$ in a uniform radial magnetic field, $B_{r}$. The coil is positioned in the center of and co-planar with the radial magnetic field. The term $\ell$ can be identified with the coil circumference times the number of turns on the coil. Imagine now the coil traversing the radial field in an axial direction at a given velocity, $v_{z}$. An emf $\varepsilon$ is developed across the coil terminals and is measured in terms of a laboratory as-maintained unit of voltage. In analogy with eq. (1), its magnitude is given by

$$
\varepsilon=\varepsilon_{N B S}\left(V_{N B S} / V\right)=N 2 \pi a_{o} B_{r} v_{z}
$$

Here, $V_{N B S}$ is the NBS as-maintained unit of voltage, $V$ the absolute or SI volt, and $\varepsilon_{N B S}$ the induced emf measured in terms of $V_{N B S}$.

Assuming in eq (4) that the velocity $v_{z}$ is measured in terms of the meter and the second, then the product of the number of turns $N$ times the circumference $2 \pi a_{o}$ times the magnitude of the magnetic field $B_{r}$ is "calibrated" and given by

$$
N 2 \pi a_{o} B_{r}=\varepsilon_{N B S}\left(V_{N B S} / V\right) / v_{z}
$$

Now imagine the same coil being suspended in the same radial magnetic field from a balance beam and carrying a current $I$ measured in terms of the NBS as maintained unit of current, $A_{N B S}$. The resulting force is then determined by use of the balance, a calibrated mass $m$, and knowledge of the local value of the gravitational acceleration, $g$. In analogy with eq(3) one obtains

$$
F=m g=N 2 \pi a_{o} B_{r} I=N 2 \pi a_{o} B_{r} I_{N B S}\left(A_{N B S} / A\right) .
$$

Using eq (5) we obtain

$$
m g v_{z}=\varepsilon_{N B S} I_{N B S}\left(V_{N B S} / V\right)\left(A_{N B S} / A\right)
$$

Noting that $V_{N B S}=A_{N B S} \Omega_{N B S}$, where $\Omega_{N B S}$ is the NBS asmaintained unit of resistance, yields finally

$$
K_{A} \equiv\left(A_{N B S} / A\right)=\left[\frac{m g v_{z}}{\varepsilon_{N B S} I_{N B S}\left(\Omega_{N B S} / \Omega\right)}\right]^{1 / 2}
$$

The ratio of the NBS ohm to the absolute ohm, $\Omega_{N B S} / \Omega$, may be obtained using the calculable cross capacitor to an accuracy of a few parts in $10^{8}$ [8]. The quantity $g$ may be obtained to the same accuracy by using an absolute gravime- 
ter. The remaining terms in the square brackets are measured during the course of the experiment. It should also be noted that eq (8) can be shown to hold when the force, velocity, and induced emf are replaced by their average values [See Appendix A] [6,7].

\section{Design}

The classical current balance configuration is inherently a good design. This configuration contains a high degree of symmetry as well as being a relatively simple geometry to construct. The current balance configuration would lend itself very nicely to the Kibble approach if it were possible to compensate the radial magnetic field component such that: (i) the force on the suspended coil is relatively independent of its axial location $(d F / d z \approx 0)$; and (ii) the force on the suspended coil is relatively independent of its own diameter $2 a_{0}\left(d F / d a_{0} \approx 0\right)$.

The reason for condition (i) is that in the first part of the experiment, the suspended coil is made to traverse the radial field axially at a constant velocity. Unless $d F / d z \approx 0$, the generated emf will be difficult to ascertain at any particular instant of time because of the response time of the suspended coil and of the detector system. This situation leads to the desirability of the condition $d F / d z=0$, particularly at the beginning and end of its travel. Condition(ii) is desirable because when the velocity is measured, the suspended coil will not have a current flowing through it, but there will be a current present when measuring the force. The heat generated by this current could change the diameter of the suspended coil by as much as $25 \mathrm{ppm} /{ }^{\circ} \mathrm{C}$. While a heat source could be used to keep the power dissipation in the suspended coil constant, it would be desirable to minimize this systematic error by careful design

With these two fundamental conditions in mind the analytical solution can begin.

The analysis of the classical current balance starts with the following equation which gives the force acting on one current loop by another current loop (see fig. 1) [9].

$$
F_{z}=\frac{I_{1} I_{2} \mathrm{zk}}{\sqrt{a_{1} a_{2}}}\left[\left(\frac{2-k^{2}}{1-k^{2}}\right) E-2 K\right] \pi .
$$

Here, $K$ and $E$ are the complete elliptic integrals of the first and second kind, respectively. The modulus $k$ is

$$
k=\left[\frac{4 a_{1} a_{2}}{z^{2}+\left(a_{1}+a_{2}\right)^{2}}\right]^{1 / 2} .
$$

After some consideration, one soon realizes that the primary magnetic field producing coils of the classical current bal-

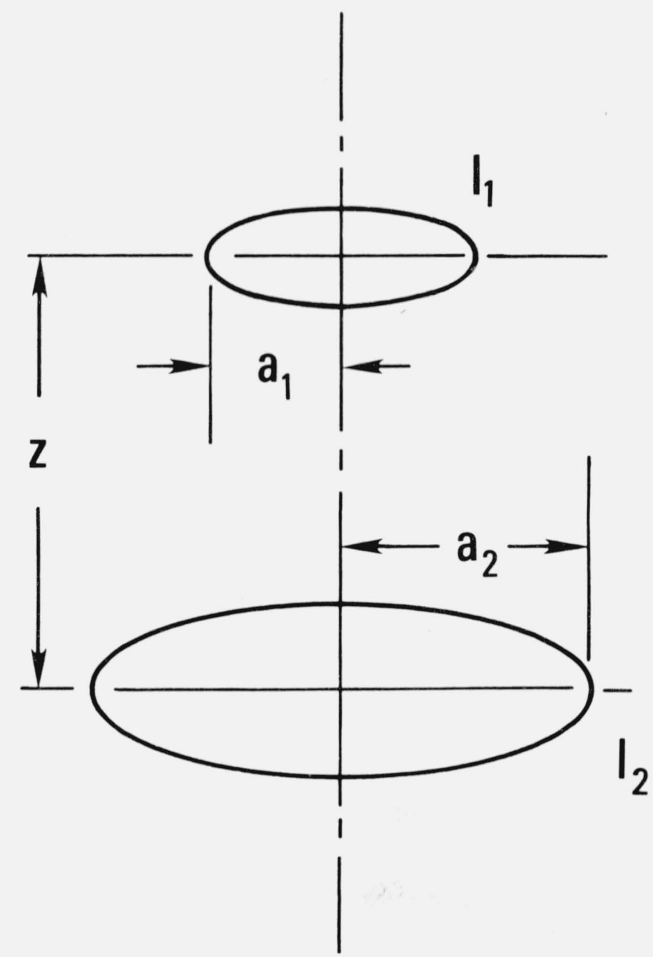

FIGURE 1. Two coaxial loops are separated by a distance $\mathrm{z}$.

Current loop 1 has a radius of $a_{1}$ and a current of $I_{1}$ : Current loop 2 has a radius of $a_{2}$ and a current of $I_{2}$.

ance cannot simultaneously meet conditions (i) and (ii) stated above. It will thus be necessary to add "compensation coils" in a configuration such as shown in figure 2 . Unfortunately, applying the force equation (9) to such a system and attempting to solve for a configuration satisfying conditions (i) and (ii) is quite difficult. Thus it was decided that a computer would be used to examine many of the possible configurations. After some preliminary calculations the following possibilities were examined, assuming a fixed $a_{2}$ :

$$
\begin{aligned}
& 0<a_{1} \leqslant a_{2} \\
& 0<z_{1} \text { to greater than } z_{2} \\
& 0.2 a_{2}<z_{2}<2 a_{2} \\
& -10<\left(I_{1} / I_{2}\right)<10
\end{aligned}
$$

The shaded region of figure 2 was the volume studied to see if solutions consistent with conditions (i) and (ii) existed. The suspended coil of diameter $2 a_{0}$ would be located somewhere in this region.

The immediate results indicated, as would be expected, that there is not a unique solution but that many solutions exist.

What follows is a description of the solution considered to be the most appropriate for the task intended. For ease of discussion, $2 a_{2}$, the diameter of the outer fixed coil, was chosen to be $100 \mathrm{~cm}$ but note that once the relative values 


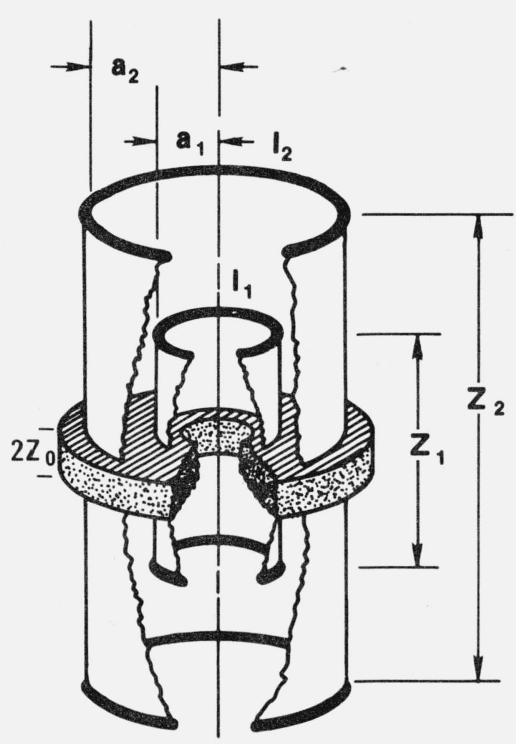

FIGURE 2. A four coil system consists of two coil pairs. The coil pairs are coaxial and concentric. The magnetic fields in the axial direction produced by the upper coils in each pair are opposed by the axial direction magnetic fields produced by the lower coils in each pair. The resultant magnetic field in the shaded region is almost entirely directed in the radial direction. The computer searched the shaded volume for a region where the magnetic field behavior was such that when a current loop, coaxial with the coil pairs, was suspended in the region, the current loop would have a constant vertical force exerted on it regardless of its diameter or vertical position.

for all coil diameters as well as the separation of the fixed coils are determined, the entire apparatus may be scaled up or down without affecting the vertical force exerted on the suspended coil, provided that both the number of windings and the current in the windings remain constant. On the scale chosen, the diameter of the suspended coil at which favorable solutions were found is around $78 \mathrm{~cm}$.

Figure 3 shows the variation in force on the suspended coil as a function of its vertical position for various separations of the coil pairs. All solutions show a similar behavior. The $\mathrm{z}$ axis is the vertical displacement of the suspended coil about the mid-plane $(z=0)$ of the fixed coil assembly. The change in force on the suspended coil relative to that at the mid-plane is designated $\Delta F$. The positive position at which $\Delta F / d z=0$ is defined to be $z_{0}$. Thus, the dashed line is the locus of the points $z_{0}$. The change in force on the suspended coil at $z_{0}$ relative to the force at the mid-plane is designated $\Delta F_{0}$. The $\Delta F_{0}$ and $z_{0}$ values are determined by separation of the two pairs of fixed coils and can be adjusted by changing $z_{1}$ and $z_{2}$, the separation of the fixed coil pairs. The curves a through e show the $\Delta F$ as a function of $z$. From one curve to the next (a to e), $z_{1}$ and $z_{2}$, the separation of the fixed coils, have been increased in the manner indicated. From eq (3), we can see that the change in emf generated for a constant velocity is proportional to $\Delta F$. It is desirable to have $\Delta F$ and $\Delta \varepsilon$ as small as possible for accurate measurements of $F_{\text {ave }}$ and $\varepsilon_{\text {ave }}$. At the same time, it is desirable to have large $z_{0}$,

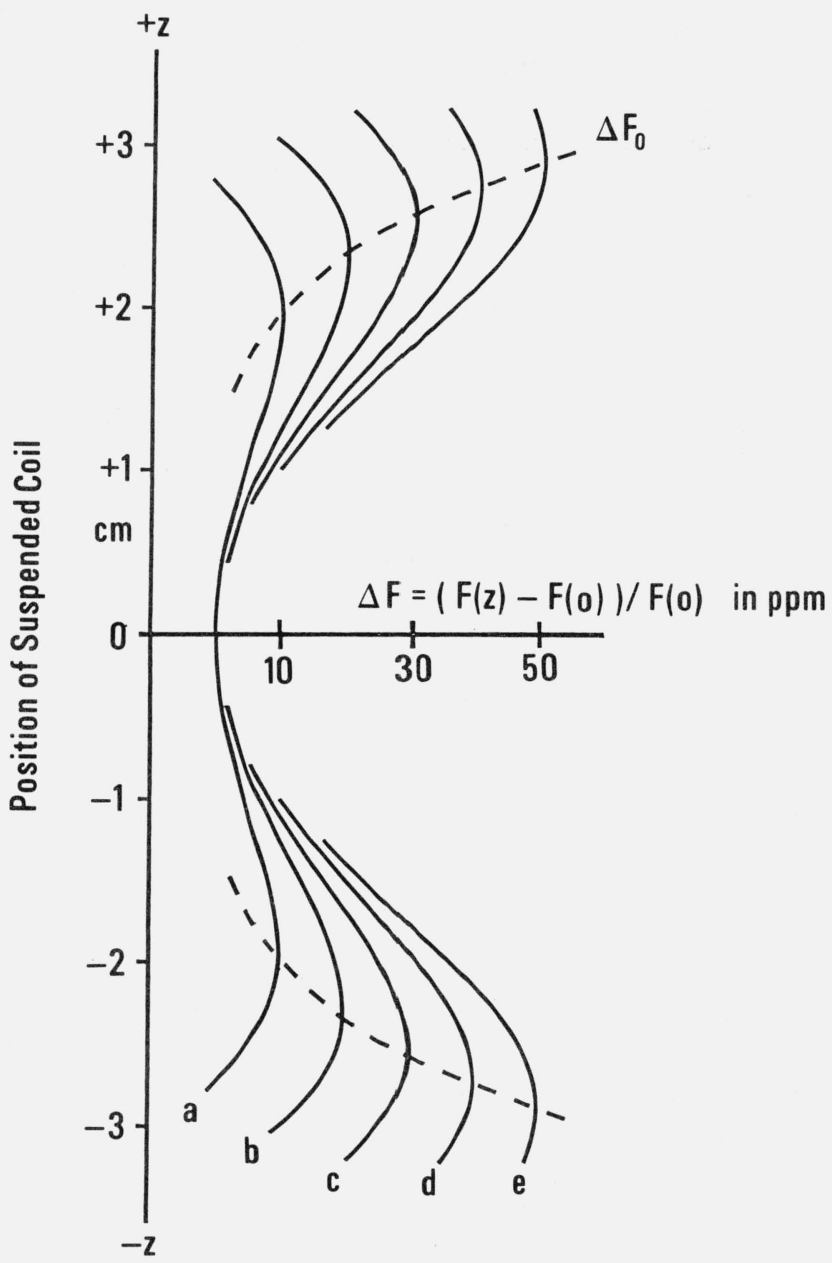

FIGURE 3. The change in vertical force on the suspended coil is a function of its vertical displacement about the mid-plane of the fixed coil system.

The object of the fixed coil design is to allow a maximum suspended coil displacement for the least amount of total force change. The dotted line connects the locus of points at which $\Delta F / d z=0$. While $\Delta F_{0}$ is the force change in ppm at the point where $\Delta F / d z=0$ referenced to the mid-plane force.

The $\Delta F$ as a function of $z$ was calculated for a coil system where $a_{1}=11 \mathrm{~cm}, a_{2}=50 \mathrm{~cm}, I_{1} / I_{2}=$ $5.5438, a_{0}$ (the radius of the suspended coil) $=39 \mathrm{~cm}$.

\begin{tabular}{cccc}
\hline \hline Curve & $\Delta F_{0}(\mathrm{ppm})$ & $\boldsymbol{z}_{\mathbf{2}}(\mathrm{cm})$ & $\mathrm{z}_{1}(\mathrm{~cm})$ \\
\hline a & 10 & 87.6836 & 51.5370 \\
$\mathrm{~b}$ & 20 & 87.8432 & 51.6166 \\
$\mathrm{c}$ & 30 & 87.9702 & 51.6796 \\
$\mathrm{~d}$ & 40 & 88.0794 & 51.7336 \\
$\mathrm{e}$ & 50 & 88.1768 & 51.7816 \\
\hline
\end{tabular}

since the total coil displacement $2 z_{0}$ must be measured accurately. Therefore we must compromise between the desire for a large $z_{0}$ and a small $\Delta F_{0}$.

Figure 4a shows the solutions for the separation of the fixed coils. The abscissa is the diameter of the inner fixed coil $2 a_{1}$. The ordinate on the left side is the separation of the outer coils $z_{2}$ and the ordinate on the right side is the separate of the inner fixed coils $z_{1}$. For $2 a_{1}$ greater than 24.0 $\mathrm{cm}$, there is a gradual deterioration of the features desirable 


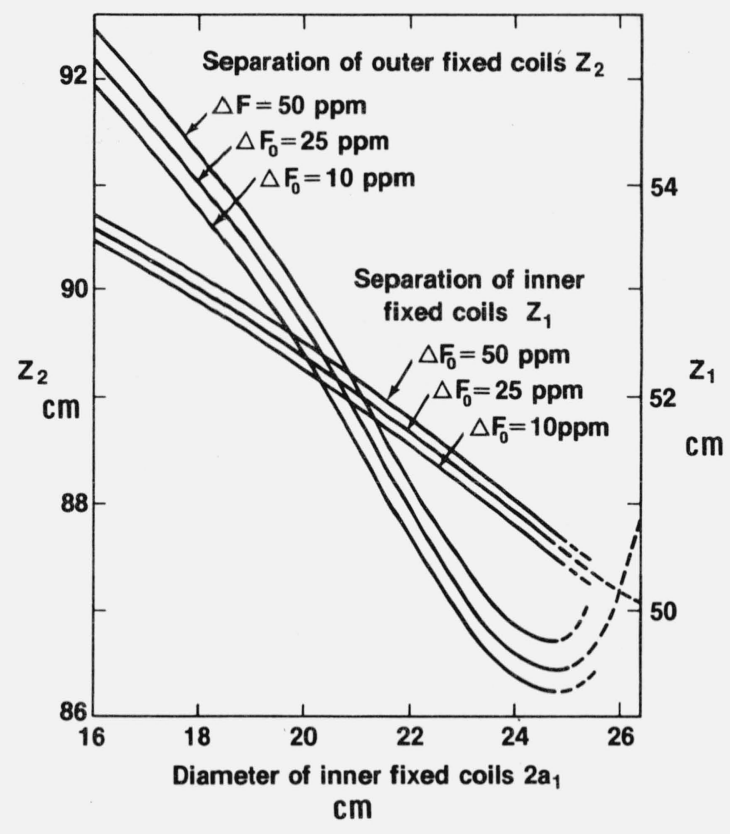

FIGURE 4a. For a fixed $2 \mathrm{a}_{2}$, the diameter of the outer fixed coils, the separation of the fixed inner and outer coils $\mathrm{z}_{1}$ and $\mathrm{z}_{2}$ can be plotted as a function of $2 \mathrm{a}_{1}$, the diameter of the inner fixed coils.

For a $2 a_{1}$ greater than about $25 \mathrm{~cm}, z_{2}$, the separation of the outer fixed coils, increases very rapidly.

for a working current balance. Specifically, the force becomes increasingly sensitive to changes in the diameter of the suspended coil. Three curves are shown for each coil separation corresponding to a $\Delta F_{0}$ of 10,25 and $50 \mathrm{ppm}$ (as labeled). The next figure (fig. $4 \mathrm{~b}$ ) shows the working length or total usable distance $2 z_{0}$ that the suspended coil can travel. The abscissa is $2 a_{1}$, the diameter of the inner fixed coils. The total usable length is shown for a $\Delta F_{0}=10,25$, and $50 \mathrm{ppm}$. The usable length decreases slightly for an increase in $2 a_{1}$ but the change is really insignificant. For a further increase in $\Delta F_{0}$, the total usable length of travel of the suspended coil does not increase significantly.

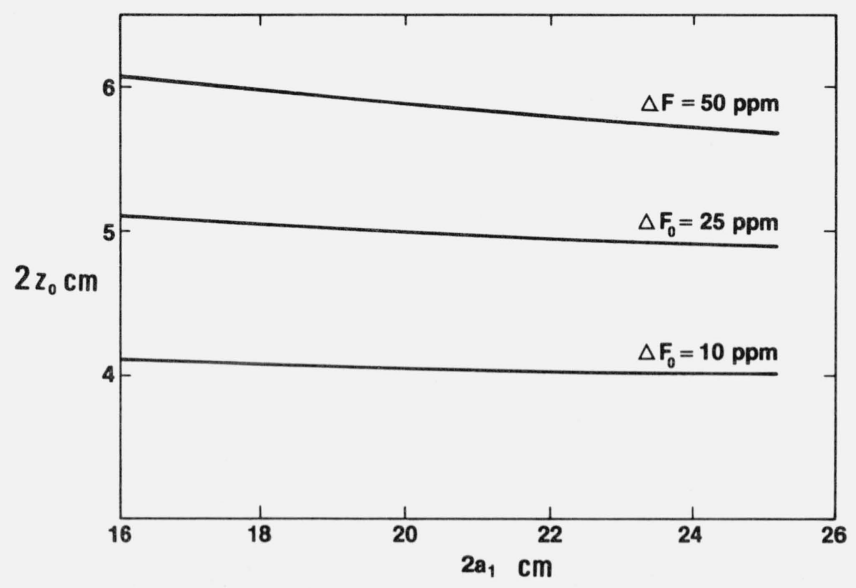

FIGURE $4 \mathrm{~b}$. The total measurable length $2 \mathrm{z}_{0}$ as a function of $\Delta \mathrm{F}_{0}$ is plotted against $2 \mathrm{a}_{1}$, the diameter of the inner fixed coils.

While there is a decrease in $2 z_{0}$ for an increase in $2 a_{1}$, the decrease is not significant.
For ease in operation, it would be desirable to have the same current passing through all of the fixed coils. It has been determined, though, that for a specific force to be exerted on the suspended coil for a given current in the fixed coils, that the number of turns of wire on each pair of fixed coils is a function of the diameter $\left(2 a_{1}\right)$ of the inner fixed coils. Figure $4 \mathrm{c}$ gives the required turns ratio $\left(N_{1} / N_{2}\right)$ of inner fixed coils to outer fixed coils as well as the number of turns required on the outside fixed coils $N_{2}$ as a function of $2 a_{1}$. The abscissa is $2 a_{1}$. The left ordinate is the turns ratio while the right ordinate is the number of turns on each outer fixed coil.

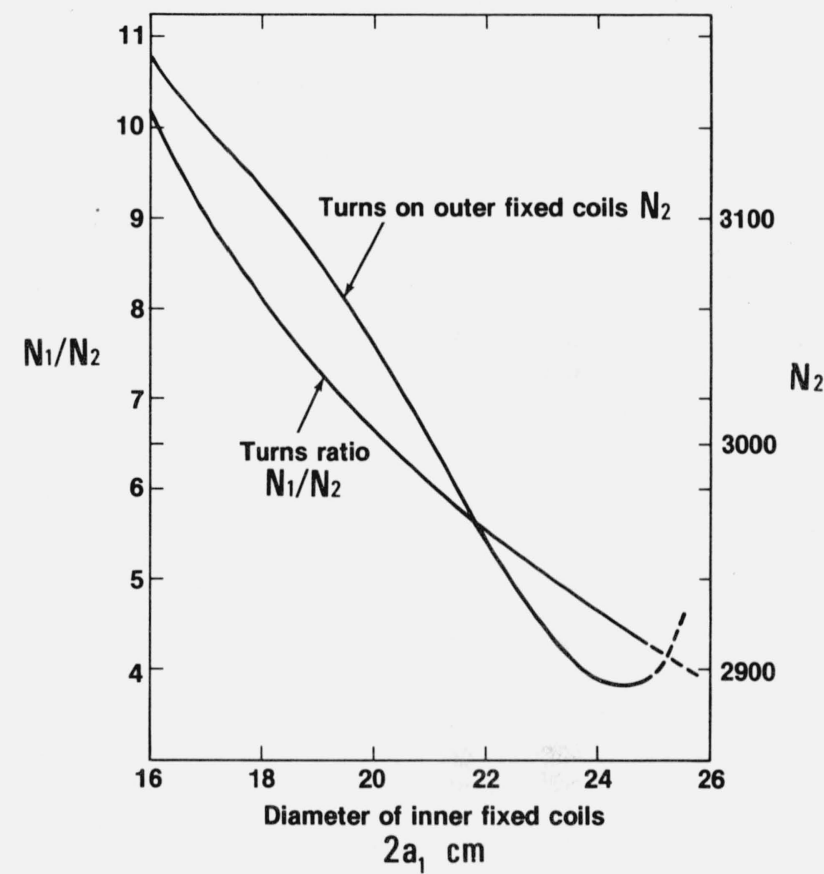

FIGURE 4c. The number of turns of wire required on each of the fixed coils are plotted with respect to $2 \mathrm{a}_{1}$, the diameter of the inner fixed coils.

The current in the wire as well as the vertical force on the suspended coil are constant. Clearly the most advantageous design would call for an inner coil diameter of about $24 \mathrm{~cm}$. For this choice the total wire necessary for both the inner and outer fixed coil is the least.

Figures $4 a, b$, and $c$ contain the necessary information required to build the field coils of a current balance. It was previously stated that a deterioration takes place for $2 a_{1}$, inner fixed coil diameter, much greater than $24 \mathrm{~cm}$. For $2 a_{1}$ less than $16 \mathrm{~cm}$, solutions continue but from figure $4 \mathrm{c}$, one can see that the number of turns on the inner fixed coils increases very rapidly without benefit; therefore it is impractical to consider a further decrease in $2 a_{1}$. On the other hand, a larger $2 a_{1}$ decreases the total number of turns on both coils. As an example, for $2 a_{1}=20 \mathrm{~cm}$, each of the outer fixed coils contains 3043 turns and the inner fixed coils contain 20,169 turns. For $2 a_{1}=24 \mathrm{~cm}$, each of the outer fixed coils contains 2895 turns and the inner fixed coils contain 13,518 turns. This corresponds to about 6000 $\mathrm{m}$ less wire. As for the current in these windings, $0.6 \mathrm{~A}$ pro- 
duces a force of $0.049 \mathrm{~N}$ on the suspended coil when the suspended coil is energized with 10 Ampere-turns (At). Correspondingly $6 \mathrm{~A}$ generates a $0.49 \mathrm{~N}$ force and $60 \mathrm{~A}$ generates $4.9 \mathrm{~N}$. Upon a current reversal of the $10 \mathrm{At}$ in the suspended coil the counter-balancing mass required is 10 , $10^{2}$, and $10^{3} \mathrm{~g}$ respectively.

\section{Coil geometry evaluation}

Figures $5 \mathrm{a}, \mathrm{b}$, and $\mathrm{c}$ show the force gradient over the area of interest where the maximum $\Delta F_{0}$ on the suspended coil is approximately 10,25 , and $50 \mathrm{ppm}$ respectively. The change in the force is plotted based on the calculated force exerted on a "test" circular filament whose diameter is allowed to expand from $2 a_{0}-2 \mathrm{~cm}(76 \mathrm{~cm})$ to $2 a_{0}+2 \mathrm{~cm}(80 \mathrm{~cm})$. The test filament is also displaced vertically about the mid-plane $(z=0)$ to $3 \mathrm{~cm}$ above and below the mid-plane $(z=+3 \mathrm{~cm}$ and $-3 \mathrm{~cm}$ ). The radial distance, $a_{0}$, on the plane corresponds to the radius of the suspended coil. The dotted line shows the path of the suspended coil as it travels vertically about $z=0$. Only the negative travel is shown since the force gradients are symmetrical about the mid-plane. The change in the peak $\Delta F_{0}$ occurs by changing only the spacings $z_{1}$ and $z_{2}$. The vertical force of course does decrease when the coil spacings are increased, about 0.6 percent from $\Delta F_{0}=10 \mathrm{ppm}$ to $\Delta F_{0}=50 \mathrm{ppm}$. This decrease can be then corrected by increasing the current in the fixed coils.

\section{Current balance specifics}

From figures $5 \mathrm{a}, \mathrm{b}$, and $\mathrm{c}$, it can be seen that the extent of available travel $2 z_{0}$ of the suspended coil varies from about $4 \mathrm{~cm}$ for a $\Delta F_{0}$ of $10 \mathrm{ppm}$ to about $6 \mathrm{~cm}$ for a $\Delta F_{0}$ of $50 \mathrm{ppm}$. This distance must be accurately measured and pretty well sets the scale of the apparatus to be built. For a total vertical travel of the suspended coil from $z=-3 \mathrm{~cm}$ to $z=+3$ $\mathrm{cm}$, an uncertainty of $0.01 \mu \mathrm{m}$ in the displacement will yield a corresponding experimental uncertainty of $0.17 \mathrm{ppm}$ for the case where $\Delta F_{0}$ is a maximum of $50 \mathrm{ppm}$.

The dimensions of the apparatus used in all of the following evaluation calculations are:

The diameter of the outer fixed coils $\left(2 a_{2}\right)$ is $1.0 \mathrm{~m}$.

The diameter of the inner fixed coils $\left(2 a_{1}\right)$ is $0.22 \mathrm{~m}$.

The diameter of the suspended coil $\left(2 a_{0}\right)$ is $0.78 \mathrm{~m}$.

The separation of the outer fixed coils $\left(z_{2}\right)$ is $0.879 \mathrm{~m}$.

The separation of the inner fixed coils $\left(z_{1}\right)$ is $0.517 \mathrm{~m}$.

The current ratio or turns ratio $\left(N_{1}\right.$ of the inner fixed coil to that of the outer fixed coils is 5.54 .

$17.75 \times 10^{4}$ Ampere-turns on the outer fixed coils and 98.32 $\times 10^{4} \mathrm{At}$ on the inner fixed coils will produce a force on the suspended coil that can be counter-balanced by a mass of 1 $\mathrm{kg}$ when the suspended coil contains $10 \mathrm{At}$.

The magnetic field of the fixed coils will generate a one volt d.c. signal across the suspenáed coil when the suspended coil containing 4081 turns traverses in the axial direction at a constant velocity of $0.5 \mathrm{~mm} / \mathrm{s}$. A current of $2.45 \mathrm{~mA}$ in such a suspended coil is required to produce a force that will support a mass of $1 \mathrm{~kg}$.

Obviously, the specifications just presented will require that the fixed coils of the current balance be superconducting. A current of $60 \mathrm{~A}$ in the fixed coils will require that there be 2960 turns on the outer fixed coils and 16,390 turns on the inner fixed coils. The fixed coils are not very unusual and should not be difficult to produce.

The suspended coil does present a problem if it is also to be at cryogenic temperatures. Since the balance will have to be at room temperature along with the standard mass, some feed-through system would have to be devised which would transfer the force on the suspended coil up to the balance arm. The suspended coil cannot be in the liquid helium but will have to be in a separate vessel and helium gas used to carry away the heat. An alternate method is to have the suspended coil in its own special dewar with that dewar suspended from the balance beam. The first method allows the laser interferometer optical components to be directly mounted onto the coil forms. The index of refraction, however, will have to be continuously monitored. The second method will have the interferometer optical components attached to the outside of the dewar. This will introduce an additional uncertainty in the measurements of length. The index of refraction however can be more easily monitored.

A third method is to have the suspended coil at room temperature. Certainly a room temperature suspended coil greatly reduces the mechanical difficulties and should be seriously considered.

The total length of wire needed on the suspended coil is $10,000 \mathrm{~m}$. With AWG 36 gauge oxygen free high conductivity copper wire, the resistance is $13.6 \mathrm{k} \Omega$. Measuring the generated d.c. emf with a $50 \mathrm{~Hz}$ band width limits the accuracy to about $0.1 \mathrm{ppm}$ due to Johnson noise. However integrating the voltage over a $120 \mathrm{~s}$ period limits the experimental accuracy to about $0.002 \mathrm{ppm}$. When weighing, the current will produce about $90 \mathrm{~mW}$ of heating. This power will increase the temperature of the coil by less than $0.03{ }^{\circ} \mathrm{C}$. Such a room temperature coil will have a mass of about 2.2 $\mathrm{kg}$ assuming the copper wire to be wound on a bobbin of low-expansion glass. Such a system is very appealing to start with and exhibits the possibility of reducing the uncertainty in $K_{A}$ by an order of magnitude immediately. On the other hand, one can imagine the ultimate current balance having the suspended coil at cryogenic temperatures. Such a system would compare the generated emf directly to the output voltage of a Josephson junction at the $5 \mathrm{mV}$ level. Whereas 


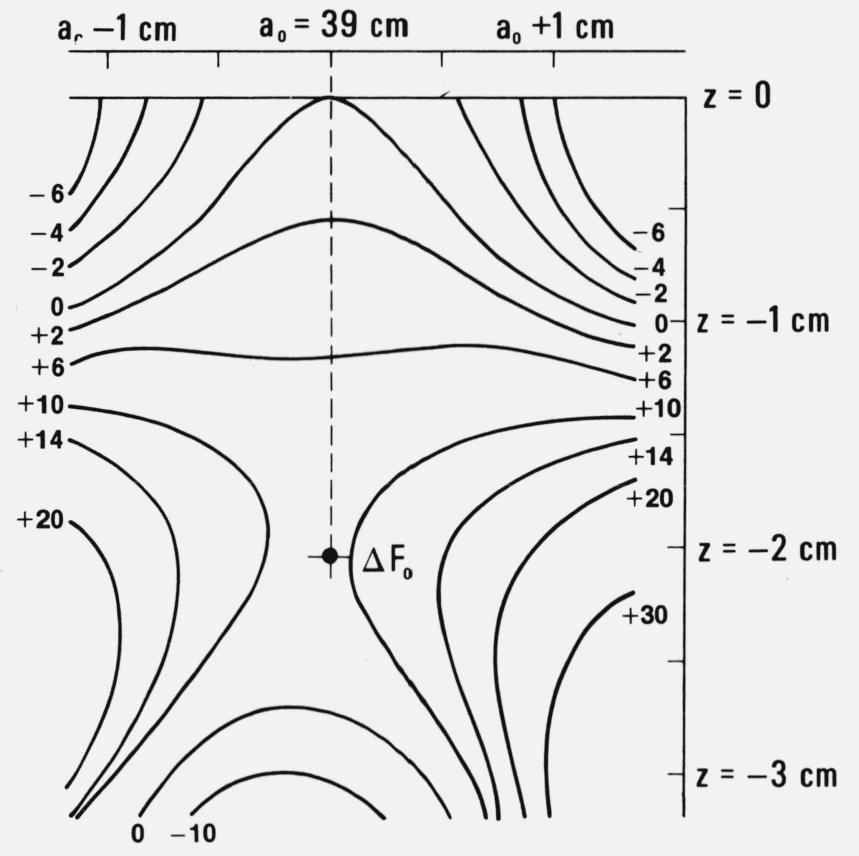

$\triangle F$ in ppm

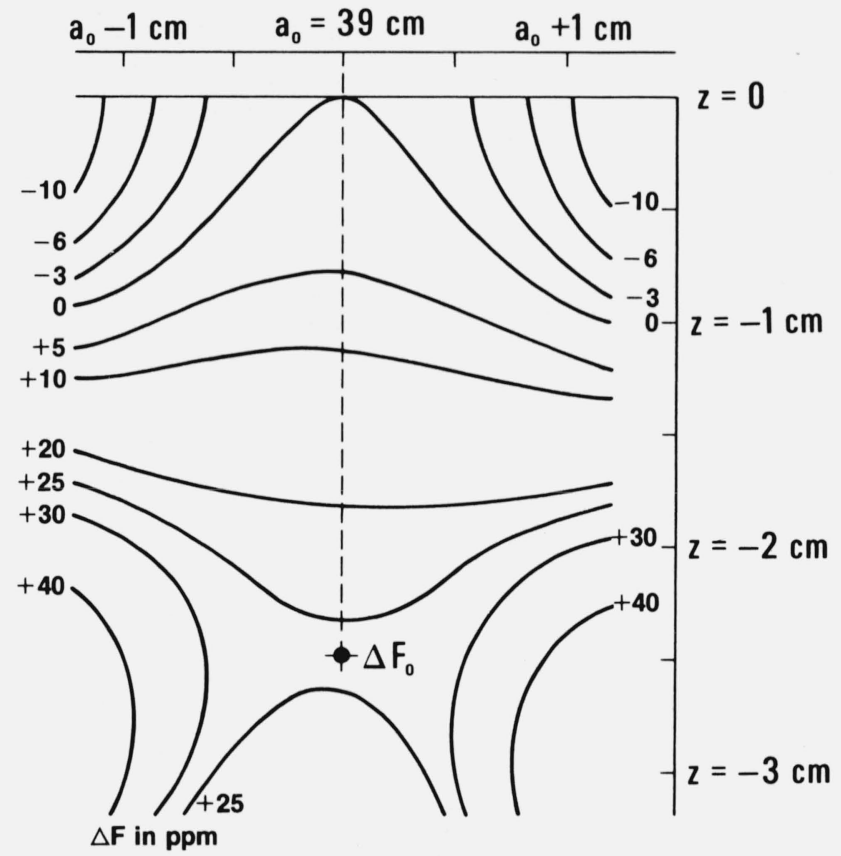

(5b)

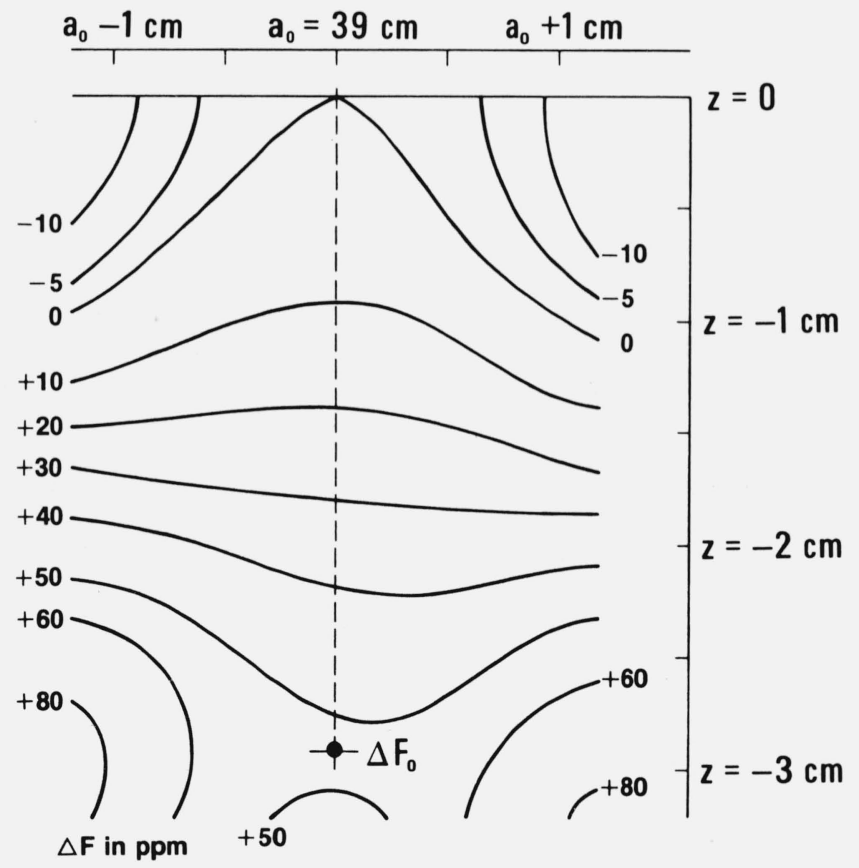

(5c)

FIGURE 5. Figures $a, b$, and c show the computed force on a test current filament, coaxial with the fixed coils, whose radius $a_{0}$ is permitted to change'from 38 to $40 \mathrm{~cm}$.

This test filament is at the same time displaced vertically from the mid-plane $(z=0)$ to + and -3 $\mathrm{cm}$. At the mid-plane position the vertical force on the current filament is a maximum at $a_{0}=39$ $\mathrm{cm}$. For off mid-plane positions, with $a_{0}=39 \mathrm{~cm}$, the force increases to a maximum at $z_{0}$ (indicated by $\Delta F_{0}$ ). At this point the $\Delta F$ is at a minimum for changes in $a_{0}$. The figure 5 mappings were made for a $2 a_{1}$ of about $20 \mathrm{~cm}$ but the results are very similar for all $2 a_{1}$ which gave a useful solution. 
the room temperature coil has an axial velocity of $0.5 \mathrm{~mm} / \mathrm{s}$ to generate $1 \mathrm{~V}$ with a sampling time of $120 \mathrm{~s}$, the all-cryogenic system would have an axial velocity of $0.0025 \mathrm{~mm} / \mathrm{s}$ and a sampling time of $24 \times 10^{3} \mathrm{~s}$, assuming all other factors remained constant.

\section{Effect of Possible Coil Misalignments}

It is generally easier to compute results for perfect coils ideally aligned than it is to produce such an assembly. The following are the computed results for various misalignments.

(1) The change in the vertical force on the suspended coil for the case where the midplanes of the inner and outer coil pairs are not coincident turns out to be the most critical (see table la). Table la shows the change in force in ppm on the suspended coil at the midplane $z=0$ for misalignments in the axial direction of $0,0.004,0.008,0.012,0.016$, and 0.020 $\mathrm{cm}$. For the suspended coil at vertical positions of $1,2,3$, and $4 \mathrm{~cm}$ away from the midplane, the change in force in ppm is given as the change in force from the midplane position for that particular misalignment. This misalignment superimposes a linear force gradient on the ideal force gradient in the left column. The force at the mid-plane changes a very small amount as indicated at the position $z=0$ for the displacements of 0.004 , etc. For the off mid-plane positions of the suspended coil where $z=1,2$ etc., the gradients $\Delta F$ from the mid-plane force become quite asymmetric rather rapidly. Fortunately, this misalignment is one which can be measured and perhaps corrected for using techniques from the authors' previous experience in the gamma-p experiment. $(10,11)$.

(2) Table lb shows the change in the vertical force on the suspended coil for the case where the inner fixed coil assembly is not coaxial with the outer and suspended coils (radial misalignment). The force gradients are symmetrical about $z=0$. The change in force at the mid-plane $(z=0)$ is with respect to the force for perfect alignment while the change in force for positions off the mid-plane are with respect to the mid-plane force for that particular misalignment.

TABLE 1a. The variation in the vertical force on the suspended coil for the case where either the inner or outer fixed coil assemblies are misaligned in the axial direction. The quantity given is $\Delta F$ in ppm. The $\Delta F$ at $z=0$ is referenced to the force for perfect alignment, while the $\Delta F$ at vertical displacements of the suspended coil is referenced to the force at the mid-plane for that particular misalignment

\begin{tabular}{|c|c|c|c|c|c|c|}
\hline \multirow{2}{*}{$\begin{array}{l}\text { Displacement } \\
\mathrm{Z}(\mathrm{cm}) \text { of } \\
\text { suspended coil }\end{array}$} & \multicolumn{6}{|c|}{ Misalignment $(\mathrm{cm})$ in axial direction } \\
\hline & 0.000 & 0.004 & 0.008 & 0.012 & 0.016 & 0.020 \\
\hline+4 & -42.7 & -56.0 & -69.4 & -82.7 & -96.1 & -109.41 \\
\hline+3 & +18.68 & +8.93 & -0.817 & -10.57 & -20.32 & -30.07 \\
\hline+2 & +21.99 & +15.62 & +9.24 & +2.86 & -3.51 & -9.89 \\
\hline+1 & +7.56 & +4.41 & +1.26 & -1.89 & -5.04 & -8.19 \\
\hline 0 & 0 & -0.006 & -0.025 & -0.056 & -0.10 & -0.157 \\
\hline-1 & +7.56 & +10.716 & +13.87 & +17.02 & +20.17 & +23.32 \\
\hline-2 & +21.99 & +28.37 & +34.74 & +41.12 & +47.49 & +53.86 \\
\hline-3 & +18.68 & +28.42 & +38.17 & +47.91 & +57.65 & +67.39 \\
\hline-4 & -42.7 & -29.4 & -16.0 & -2.719 & +10.61 & +23.93 \\
\hline
\end{tabular}

TABLE $1 \mathrm{~b}$. The variation in the vertical-force on the suspended coil for the case where the inner fixed coil assembly is misaligned in the radial direction. The quantity given is $\Delta F$ in ppm. The $\Delta F$ at $z=0$ is referenced to the force for perfect alignment, while the $\Delta F$ at vertical displacements of the suspended coil is referenced to the force at the mid-plane for that particular misalignment.

\begin{tabular}{|c|c|c|c|c|c|c|}
\hline \multirow{2}{*}{$\begin{array}{l}\text { Displacement } \\
\mathrm{Z}(\mathrm{cm}) \text { of } \\
\text { suspended coil }\end{array}$} & \multicolumn{6}{|c|}{ Misalignment $(\mathrm{cm})$ in radial direction } \\
\hline & 0.0 & 0.4 & 0.8 & 1.2 & 1.6 & 2.0 \\
\hline+4 & -42.7 & -36.9 & -19.6 & +9.33 & +49.9 & +102.3 \\
\hline+3 & +18.68 & +21.97 & +31.84 & +48.33 & +71.47 & +101.3 \\
\hline+2 & +21.99 & +23.47 & +27.90 & +35.29 & +45.67 & +59.0 \\
\hline+1 & +7.56 & +7.94 & +9.05 & +10.91 & +13.52 & +16.88 \\
\hline 0 & 0 & +31.4 & & +282 & & +780 \\
\hline
\end{tabular}

Note: $\Delta \mathrm{F}$ is symmetrical about the midplane of the coil system. 
(3) Table 1c shows the change in vertical force on the suspended coil for the case where the outer fixed coil assembly is not coaxial with the inner and suspended coils (radial misalignment). The change in force at the mid-plane $(z=0)$ is with respect to the force for perfect alignment while the change in force for positions off the mid-plane are with respect to the mid-plane force for that particular misalignment.

(4) Table 1d shows the change in vertical force on the suspended coil for the case where the suspended coil is not coaxial with the inner and outer coils (radial misalignment). It should be noted that the amount of misalignment given in tables $1 \mathrm{~b}-1 \mathrm{~d}$ is 100 times greater than the axial misalignment of table la. The change in force on the suspended coil at the mid-plane for the outer fixed coils radially misaligned is opposite the change in force for the case where the inner fixed coils are radially misaligned. The change in force at the mid-plane $(z=0)$ is with respect to the force for perfect alignment while the change in force for positions off the mid-plane are with respect to the mid-plane force for that particular misalignment. Even though the force change is substantial (some $30 \mathrm{ppm}$ for a $4 \mathrm{~mm}$ displacement), there is very little change in the force gradient for the suspended coil being off the mid-plane.
(5) Table le shows the change in the vertical force on the suspended coil for the case where the suspended coil is tilted about the center of the system. The change in force at the mid-plane $(z=0)$ is with respect to the force for perfect alignment while the change in force for positions off the mid-plane are with respect to the mid-plane force for that particular misalignment.

(6) Table lf shows the change in the vertical force on the suspended coil for the case where the outer fixed coil assembly is tilted about the center of the system. The change in force at the mid-plane $(z=0)$ is with respect to the force for perfect alignment while the change in force for positions off the mid-plane are with respect to the mid-plane force for that particular misalignment.

(7) Table $1 \mathrm{~g}$ shows the change in the vertical force on the suspended coil for the case where the inner fixed coil assembly is tilted about the center of the system. The change in force at the mid-plane $(z=0)$ is with respect to the force for perfect alignment while the change in force for positions off the mid-plane are with respect to the mid-plane force for that particular misalignment.

TABLE 1c. The variation in the vertical force on the suspended coil for the case where the outer fixed coil assembly is misaligned in the radial direction. The quantity given is $\Delta F$ in ppm. The $\Delta F$ at $z=0$ is referenced to the force for perfect alignment, while the $\Delta F$ at vertical displacements of the suspended coil is referenced to the force at the mid-plane for that particular misalignment.

\begin{tabular}{c|c|c|c|r|r|r}
\hline \hline \multirow{2}{*}{$\begin{array}{c}\text { Displacement } \\
\text { Z (cm) of } \\
\text { suspended coil }\end{array}$} & \multicolumn{5}{|c}{ Misalignment (cm) in radial direction } \\
\cline { 2 - 6 }+4 & 0.0 & 0.4 & 0.8 & 1.2 & 1.6 & -52.8 \\
+3 & -42.7 & -42.3 & -45.2 & -48.4 & -58.5 \\
+2 & +18.68 & +18.32 & +17.26 & +15.48 & +12.99 \\
+1 & +21.99 & +21.83 & +21.36 & +20.57 & +19.47 \\
0 & +7.56 & +7.53 & +7.41 & +7.21 & +6.78 \\
+18.04 & +6.58 \\
\hline
\end{tabular}

Note: $\Delta \mathbf{F}$ is symmetrical about the midplane of the coil system.

TABLE 1d. The variation in the vertical force on the suspended coil for the case where the suspended coil is misaligned in the radial direction. The quantity given is $\Delta F$ in ppm. The $\Delta F$ at $z=0$ is referenced to the force for perfect alignment, while the $\Delta F$ at vertical displacements of the suspended coil is referenced to the force at the mid-plane for that particular misalignment.

\begin{tabular}{|c|c|c|c|c|c|c|}
\hline \multirow{2}{*}{$\begin{array}{c}\text { Displacement } \\
\mathrm{Z}(\mathrm{cm}) \text { of } \\
\text { suspended coil }\end{array}$} & \multicolumn{6}{|c|}{ Misalignment $(\mathrm{cm})$ in radial direction } \\
\hline & 0.0 & 0.4 & 0.8 & 1.2 & 1.6 & 2.0 \\
\hline+4 & -42.7 & -37.6 & -22.1 & +3.66 & +39.9 & +86.6 \\
\hline+3 & +18.68 & +21.6 & +30.4 & +45.1 & +65.8 & +92.5 \\
\hline+2 & +21.99 & +23.3 & +27.3 & +33.9 & +43.2 & +55.1 \\
\hline+1 & +7.56 & +7.90 & +8.89 & +10.5 & +12.9 & +15.9 \\
\hline 0 & 0 & -0.67 & -2.75 & -6.48 & -12.3 & -20.6 \\
\hline
\end{tabular}

Note: $\Delta \mathrm{F}$ is symmetrical about the midplane of the coil system. 
(8) Table $\mathrm{lh}$ shows the change in the vertical force on the suspended coil for the case where both fixed coil assemblies are tilted as a unit about the center of the system. The change in force at the mid-plane $(z=0)$ is with respect to the force for perfect alignment while the change in force for positions off the mid-plane are with respect to the mid-plane force for that particular misalignment.

Of all the misalignments, the most critical is that where the midplanes of the fixed coil pairs do not coincide. The least critical alignment apparent in the vertical force on the suspended coil is where the suspended coil is either radially or angularly misaligned.

TABLE le. The variation in the vertical force on the suspended coil for the case where the suspended coil is tilted about the center of the system. The quantity given is $\Delta F$ in ppm. The $\Delta F$ at $z=0$ is referenced to the force for perfect alignment, while the $\Delta F$ at vertical displacements of the suspended coil is referenced to the force at the mid-plane for that particular misalignment.

\begin{tabular}{c|c|c|c|c|c|c|c|c|c|c}
\hline \hline \multirow{2}{*}{$\begin{array}{c}\text { Displacement } \\
\begin{array}{c}\text { Z(cm) of } \\
\text { suspended coil }\end{array}\end{array}$} & 0.0 & 0.2 & 0.4 & 0.6 & 0.8 & 1.0 & 2.0 & 4.0 & 6.0 & 8.0 \\
\cline { 2 - 11 } & & & & & & & & & & \\
\hline+4 & -42.7 & -43.1 & -44.9 & -47.9 & -52.1 & -57.5 & -102.0 & -283.0 & $\ldots \ldots \ldots$ & $\ldots \ldots \ldots$ \\
+3 & +18.68 & +18.28 & +17.28 & +15.56 & +13.20 & +10.12 & -15.45 & -118.0 & -290.0 & $\ldots \ldots \ldots$ \\
+2 & +21.99 & +21.61 & +21.16 & +20.41 & +19.35 & +17.97 & +6.51 & -39.4 & -116.0 & -225.0 \\
+1 & +7.56 & +7.41 & +7.30 & +7.11 & +6.85 & +6.50 & +3.62 & -7.90 & -27.2 & -54.0 \\
0 & 0 & +0.0076 & +0.30 & +0.67 & +1.18 & +1.85 & +6.76 & +16.4 & -3.47 & -108.0 \\
\hline
\end{tabular}

Note: $\Delta \mathrm{F}$ is symmetrical about the midplane of the coil system.

TABLE lf. The variation in the vertical force on the suspended coil for the case where the outer fixed coil assembly is tilted about the center of the system. The quantity given is $\Delta F$ in ppm. The $\Delta F$ at $z=0$ is referenced to the force for perfect alignment, while the $\Delta F$ at vertical displacements of the suspended coil is referenced to the force at the mid-plane for that particular misalignment.

\begin{tabular}{c|c|c|c|r|r|r}
\hline \hline Displacement & \multicolumn{7}{|c}{ Tilt in degrees } \\
$\begin{array}{c}\text { Z (cm) of } \\
\text { suspended coil }\end{array}$ & 0.0 & 0.2 & 0.4 & 0.6 & 0.8 & 1.0 \\
\cline { 2 - 7 } & -42.7 & -42.6 & -42.6 & -42.7 & -42.8 & -42.9 \\
+4 & +18.68 & +18.56 & +18.54 & +18.49 & +18.51 & +18.43 \\
+3 & +21.99 & +21.71 & +21.78 & +21.69 & +21.76 & +21.72 \\
+2 & +7.56 & +7.40 & +7.45 & +7.38 & +7.45 & +7.44 \\
+1 & 0 & -1.39 & -5.75 & -12.94 & -23.08 & -36.09 \\
0 & \multicolumn{7}{|c|}{}
\end{tabular}

Note: $\Delta F$ is symmetrical about the midplane of the coil system.

TABLE 1g. The variation in the vertical force on the suspended coil for the case where the inner fixed coil is tilted about the center of the system. The quantity given is $\Delta F$ in ppm. The $\Delta F$ at $z=0$ is referenced to the force for perfect alignment, while the $\Delta F$ at vertical displacements of the suspended coil is referenced to the force at the mid-plane for that particular misalignment.

\begin{tabular}{c|c|c|c|c|c|c}
\hline \hline Displacement & \multicolumn{7}{|c}{ Tilt in degrees } \\
$\begin{array}{c}\mathrm{Z}(\mathrm{cm}) \text { of } \\
\text { suspended coil }\end{array}$ & 0.0 & 0.2 & 0.4 & 0.6 & 0.8 & 1.0 \\
\cline { 2 - 7 } & -42.7 & -43.1 & -44.9 & -48.0 & -52.0 & -57.4 \\
+4 & +18.68 & +18.28 & +16.80 & +15.87 & +18.08 & +10.53 \\
+3 & +21.99 & +21.93 & +21.28 & +20.54 & +19.65 & +18.42 \\
+2 & +7.56 & +7.34 & +7.24 & +7.09 & +6.88 & +6.82 \\
+1 & 0 & -4.50 & -18.1 & -41.1 & -73.2 & -114.7 \\
0 & \multicolumn{10}{|c|}{}
\end{tabular}

Note: $\Delta \mathrm{F}$ is symmetrical about the midplane of the coil system. 
TABLE lh. The variation in the vertical force on the suspended coil for the case where the entire fixed coil assembly is tilted about the center of the system. The quantity given is $\Delta F$ in ppm. The $\Delta F$ at $z=0$ is referenced to the force for perfect alignment, while the $\Delta F$ at vertical displacements of the suspended coil is referenced to the force at the mid-plane for that particular misalignment.

\begin{tabular}{c|c|c|c|c|c|c}
\hline \hline \multirow{2}{*}{$\begin{array}{c}\text { Displacement } \\
\text { Z(cm) of } \\
\text { suspended coil }\end{array}$} & 0.0 & 0.2 & 0.4 & 0.6 & 0.8 \\
\cline { 2 - 6 }+4 & -42.7 & -43.2 & -44.9 & -48.1 & -52.3 \\
+3 & +18.68 & +18.20 & +16.71 & +15.73 & +12.96 \\
+2 & +21.99 & +21.87 & +21.29 & +20.46 & +57.8 \\
+1 & +7.56 & +7.30 & +7.25 & +7.03 & +10.33 \\
0 & 0 & -5.90 & -23.86 & -54.08 & -96.88 \\
\hline
\end{tabular}

Note: $\Delta \mathrm{F}$ is symmetrical about the midplane of the coil system.

\section{Non Vertical Forces}

Perhaps even more important than the change in vertical forces for coil misalignment, is the unmeasured horizontal forces acting on the suspended coil. In the calibrating part (the measuring of the generated emf) of the experiment, a horizontal force translates into an error emf if there is any horizontal velocity. This "horizontal" emf adds directly to the vertical emf and becomes an error in the force calibration unless it is properly accounted for.

Table 2a shows the horizontal force on the suspended coil for a misalignment in the radial direction of the suspended coil and for vertical positions of the suspended coil up to $z$ $=4 \mathrm{~cm}$ from the mid-plane of the system. The horizontal force is an odd function about the mid-plane. The forces given here are in parts per million (ppm) of the vertical force. The sign indicates direction i.e. if negative, the force is away from the central position, positive is a restoring force. Of course if the direction of current is reversed in the suspended coil then the direction of all forces is reversed.
Table $2 b$ is a tabulation of horizontal forces for the case where the suspended coil is tilted. Results are shown for tilts up to 2 degrees. The force is perpendicular to the axis of rotation of the coil. The horizontal force is given in ppm of the vertical force for perfect alignment.

The classical current balance configuration has the characteristic that when there is just angular (tilt) misalignment, there is no torque on the suspended coil at the mid-plane. Table 3a summarizes the torques present on the suspended coil when the only misalignment is a tilt over the coil range of vertical displacement of the suspended coil from $z=+4$ $\mathrm{cm}$ to $-4 \mathrm{~cm}$. The values given are in units of length $(\mu \mathrm{m})$. The torque is normalized to the vertical force of the perfectly aligned system.

Table $3 \mathrm{~b}$ shows the torque for the case where the inner fixed coil pair is tilted. Table $3 \mathrm{c}$ shows the torque for the case where the outer fixed coil pair is tilted. These torques are opposite and tend to cancel when it is the entire fixed coil assembly that is tilted.

TABLE 2a. Ratio of horizontal force to vertical force (in ppm) on the suspended coil for the case where the suspended coil is misaligned in the radial direction.

\begin{tabular}{|c|c|c|c|c|c|c|c|c|c|c|}
\hline \multirow{2}{*}{$\begin{array}{l}\text { Displacement } \\
\mathrm{Z}(\mathrm{cm}) \text { of } \\
\text { suspended coil }\end{array}$} & \multicolumn{10}{|c|}{ Misalignment $(\mathrm{cm})$ in the radial direction } \\
\hline & 0.0 & 0.1 & 0.2 & 0.3 & 0.4 & 0.5 & 0.8 & 1.2 & 1.6 & 2.0 \\
\hline+4 & 0.0 & -10.7 & -21.2 & -31.4 & -41.1 & -49.9 & -70.2 & -75.2 & -43.7 & +37.5 \\
\hline+3 & 0.0 & -2.4 & -4.7 & -6.7 & -8.2 & -9.2 & -7.2 & -12.5 & +60.4 & +146.5 \\
\hline+2 & 0.0 & +1.1 & +2.3 & +3.7 & +5.4 & +7.5 & +17.2 & +41.6 & +85.2 & +154.8 \\
\hline+1 & 0.0 & +1.4 & +2.8 & +4.4 & +6.0 & +7.9 & +15.2 & +30.9 & +56.2 & +94.7 \\
\hline 0 & 0.0 & 0.0 & 0.0 & 0.0 & 0.0 & 0.0 & 0.0 & 0.0 & 0.0 & 0.0 \\
\hline-1 & 0.0 & -1.4 & -2.8 & -4.4 & -6.0 & -7.9 & -15.2 & -30.9 & -56.2 & -94.7 \\
\hline-2 & 0.0 & -1.1 & -2.3 & -3.7 & -5.4 & -7.5 & -17.2 & -41.6 & -85.2 & -154.8 \\
\hline-3 & 0.0 & +2.4 & +4.7 & +6.7 & +8.2 & +9.2 & +7.2 & +12.5 & -60.4 & -146.5 \\
\hline-4 & 0.0 & +10.7 & +21.2 & +31.4 & +41.1 & +49.9 & +70.2 & +75.2 & +43.7 & -37.5 \\
\hline
\end{tabular}


TABLE 2b. Ratio of horizontal force to vertical force (in ppm) on the suspended coil for the case where the suspended coil is tilted about the center of the system.

\begin{tabular}{|c|c|c|c|c|c|c|}
\hline \multirow{2}{*}{$\begin{array}{l}\text { Displacement } \\
\mathrm{Z}(\mathrm{cm}) \text { of } \\
\text { suspended coil }\end{array}$} & \multicolumn{6}{|c|}{ Tilt in degrees } \\
\hline & 0.0 & 0.4 & 0.8 & 1.2 & 1.6 & 2.0 \\
\hline $\begin{array}{r}+4 \\
+3 \\
+2 \\
+1 \\
0\end{array}$ & $\begin{array}{l}0.0 \\
0.0 \\
0.0 \\
0.0 \\
0.0\end{array}$ & $\begin{array}{l}+3.9 \\
+0.90 \\
-0.03 \\
-0.09 \\
-0.03\end{array}$ & $\begin{array}{l}+8.1 \\
+1.9 \\
-0.02 \\
-0.17 \\
-0.05\end{array}$ & $\begin{array}{c}+12.4 \\
+3.0 \\
+0.06 \\
-0.23 \\
-0.08\end{array}$ & $\begin{array}{c}+17.2 \\
+4.4 \\
+0.25 \\
-0.27 \\
-0.11\end{array}$ & $\begin{array}{c}+22.7 \\
+6.1 \\
+0.58 \\
-0.27 \\
-0.14\end{array}$ \\
\hline
\end{tabular}

Note: The force ratio is symmetrical about the midplane of the coil system.

TABLE 3a. Ratio of torque to vertical force acting on suspended coil (unit of $\mu \mathrm{m}$ ) for the case where the suspended coil is tilted about the center of the system.

\begin{tabular}{|c|c|c|c|c|c|c|c|}
\hline \multirow{2}{*}{$\begin{array}{l}\text { Displacement } \\
\mathrm{Z}(\mathrm{cm}) \text { of } \\
\text { suspended coil }\end{array}$} & \multicolumn{7}{|c|}{ Tilt in degrees } \\
\hline & 0.0 & 0.2 & 0.4 & 0.6 & 0.8 & 2.0 & 4.0 \\
\hline+4 & 0.0 & -13.8 & -27.6 & -41.4 & -55.4 & -135.8 & -264.1 \\
\hline+3 & 0.0 & -10.5 & -20.9 & -31.4 & -41.9 & -103.0 & -200.5 \\
\hline+2 & 0.0 & -7.04 & -14.1 & -21.1 & -28.1 & -69.2 & -134.8 \\
\hline+1 & 0.0 & -3.54 & -7.07 & -10.6 & -14.1 & -34.8 & -67.8 \\
\hline 0 & 0.0 & 0.0 & 0.0 & 0.0 & 0.0 & +0.0 & 0.0 \\
\hline-1 & 0.0 & +3.54 & +7.07 & +10.6 & +14.1 & +34.8 & +67.8 \\
\hline-2 & 0.0 & +7.04 & +14.1 & +21.1 & +28.1 & +69.2 & +134.8 \\
\hline-3 & 0.0 & +10.5 & +20.9 & +31.4 & +41.9 & +103.0 & +200.5 \\
\hline-4 & 0.0 & +13.8 & +27.6 & +41.4 & +55.4 & +135.8 & +264.1 \\
\hline
\end{tabular}

TABLE 3b. Ratio of torque to vertical force acting on suspended coil (unit of $\mu \mathrm{m}$ ) for the case where the inner fixed coil is tilted.

\begin{tabular}{|c|c|c|c|c|c|}
\hline \multirow{2}{*}{$\begin{array}{l}\text { Displacement } \\
\mathrm{Z}(\mathrm{cm}) \text { of } \\
\text { suspended coil }\end{array}$} & \multicolumn{5}{|c|}{ Tilt in degrees } \\
\hline & 0.0 & 2.0 & 4.0 & 6.0 & 8.0 \\
\hline+4 & 0.0 & +34.0 & +69.0 & +106.4 & +147.2 \\
\hline+3 & 0.0 & +24.1 & +49.0 & +75.6 & +104.8 \\
\hline+2 & 0.0 & +15.4 & +31.3 & +48.4 & +67.2 \\
\hline+1 & 0.0 & +7.5 & +15.2 & +23.6 & +32.8 \\
\hline 0 & 0.0 & 0.0 & 0.0 & 0.0 & 0.0 \\
\hline-1 & 0.0 & -7.5 & -15.2 & -23.6 & -32.8 \\
\hline-2 & 0.0 & -15.4 & -31.3 & -48.4 & -67.2 \\
\hline-3 & 0.0 & -24.1 & -49.0 & -75.6 & -104.8 \\
\hline-4 & 0.0 & -34.0 & -69.0 & -106.4 & -147.2 \\
\hline
\end{tabular}

TABLE 3c. Ratio of torque to vertical force acting on suspended coil (in units of $\mu \mathrm{m}$ ) for the case where the outer fixed coil is tilted.

\begin{tabular}{|c|c|c|c|c|c|}
\hline \multirow{2}{*}{$\begin{array}{l}\text { Displacement } \\
\mathrm{Z}(\mathrm{cm}) \text { of } \\
\text { suspended coil }\end{array}$} & \multicolumn{5}{|c|}{ Tilt in degrees } \\
\hline & 0.0 & 2.0 & 4.0 & 6.0 & 8.0 \\
\hline+4 & 0.0 & -169.8 & -333.2 & -483.6 & -613.6 \\
\hline+3 & 0.0 & -127.1 & -249.5 & -362.2 & -460.1 \\
\hline+2 & 0.0 & -84.6 & -166.1 & -241.3 & -306.6 \\
\hline+1 & 0.0 & -42.3 & -83.0 & -120.6 & -153.3 \\
\hline 0 & 0.0 & 0.0 & 0.0 & 0.0 & 0.0 \\
\hline-1 & 0.0 & +42.3 & +83.0 & +120.6 & +153.3 \\
\hline-2 & 0.0 & +84.6 & +166.1 & +241.3 & +306.6 \\
\hline-3 & 0.0 & +127.1 & +249.5 & +362.2 & +460.1 \\
\hline-4 & 0.0 & +169.8 & +333.2 & +483.6 & +613.6 \\
\hline
\end{tabular}


Table $3 \mathrm{~d}$ shows the torque on the suspended coil when there is a misalignment in the radial direction between the fixed coil assembly and the suspended coil. This torque is by far the largest of all the other torques calculated. The torque is relatively constant over the expected vertical positions of the suspended coil and increases fairly linearly with increased misalignment.
Most of the effects of coil misalignment are small, but they will have to be monitored and corrections will have to be applied. For the case of vertical forces, if perfect alignment is not possible, the experimental accuracy will be unaffected, as long as the misalignment does not change during the course of operation. The chance for a change in alignment can develop during the measurement of voltage.

TABLE 3d. Ratio of torque to vertical force acting on suspended coil (in units of $\mu \mathrm{m}$ ) for the case where the suspended coil is misaligned in the radial direction.

\begin{tabular}{|c|c|c|c|c|c|c|}
\hline \multirow{2}{*}{$\begin{array}{l}\text { Displacement } \\
\mathrm{Z}(\mathrm{cm}) \text { of } \\
\text { suspended coil }\end{array}$} & \multicolumn{6}{|c|}{ Tilt in degrees } \\
\hline & 0.0 & 0.2 & 0.4 & 0.6 & 0.8 & 1.0 \\
\hline+4 & 0. & 849 & 1696 & 2546 & 3396 & 4248 \\
\hline+3 & 0 & 849 & 1698 & 2548 & 3398 & 4250 \\
\hline+2 & 0 & 849 & 1698 & 2548 & 3399 & 4251 \\
\hline+1 & 0 & 849 & 1698 & 2548 & 3399 & 4250 \\
\hline 0 & 0 & 849 & 1698 & 2548 & 3398 & 4250 \\
\hline
\end{tabular}

Note: $\Delta \mathbf{F}$ is symmetrical about the midplane of the coil system.

In this instance, the suspended coil will be changing its axial position at a uniform velocity with respect to the fixed coils. This action can occur by displacing the fixed coils, by displacing the suspended coil through movement of the entire balance, or by allowing the balance to swing. Either of the three methods will require a positive servo system to either keep the velocity constant or the generated emf constant. Certainly, the swinging of the balance is the most attractive since it requires the least effort. The swinging of the balance however naturally produces a misalignment in the radial direction of the suspended coil when it travels from $+z_{0}$ to $-z_{0}$. Depending upon the length of the balance beam, though, this displacement can be kept small. For a balance beam with a half length of $40 \mathrm{~cm}$, the maximum radial displacement is about $0.11 \mathrm{~cm}$. From Table 2a, it can be found that the horizontal force is about $2.6 \mathrm{ppm}$ of the vertical force. This horizontal force results in an additional displacement of less than $1 \mu \mathrm{m}$ when the vertical distance from the knife edge to the suspended coil is $1 \mathrm{~m}$. The horizontal force produced is a negligible error in the vertical force measurement. However, this horizontal force translates into a generated emf into the suspended coil during the calibration part of the experiment and yet does not add to the vertical force. This is a potential systematic error if not properly taken into account.

The maximum calculated horizontal velocity is $3.75 \times$ $10^{-3} \mathrm{~cm} / \mathrm{s}$ as compared to the vertical velocity of $5 \times 10^{-2}$ $\mathrm{cm} / \mathrm{s}$. This will increase or decrease the emf measured at the end points by $0.195 \mu \mathrm{V}$ in one volt. If the initial velocity is negative at $z=+3$ and the $\Delta \mathrm{emf}$ is positive then the $\Delta \mathrm{emf}$ will also be positive at $z=-3$. Therefore a systematic error will result and a correction must be applied. This correction can be calculated from a measurement of the horizontal force.

\section{Conclusion}

The first part of the experiment involves calibrating the force balance through the measurement of a d.c. voltage and a velocity. Both of these measurements are difficult but possible at the $0.1 \mathrm{ppm}$ level of uncertainty.

The second part of the experiment, "weighing the force", will be relatively easy. Considering that the mass of the suspended coil will be approximately $2.2 \mathrm{~kg}$ and the vertical electromagnetic force will be counter-balanced by mass of $1.0 \mathrm{~kg}$, a weighing to $5 \times 10^{-9}(16 \mu \mathrm{g})$ will result in an uncertainty of $0.02 \mathrm{ppm}$ in the force measurement. While a guest worker at NBS, Mr. H. Nakamura of the Electrotechnical Laboratory, Japan developed a very good servo-system for the NBS current balance [10]. Mr. Nakamura's contribution will certainly allow the force to be weighed to $0.02 \mathrm{ppm}$ or better.

We hope that the apparatus outlined above (resembling fig. 6) will resolve the confusion that now exists in present determinations of $K_{A}$. The undertaking is a large scale experiment with much apparatus to be constructed. The exact time required for achieving the ultimate accuracy in the experiment is not easily determinable, nor is the ultimate ac- 


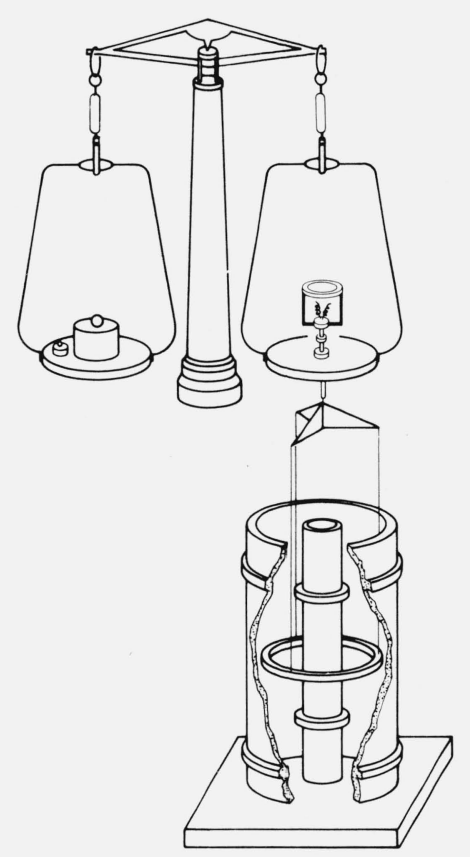

(not to scale)

FIGURE 6. The general appearance of the compensated current balance is shown.

The mass on the left scale pan of the balance is sufficient to counterbalance the mass of the suspended coil plus the downward vertical force exerted on the suspended coil. When the current in the suspended coil is reversed, then a mass $M$ is added to the right scale pan to offset the upward vertical force. Thus the mass $M$ is sufficient to counterbalance the upward vertical force plus the negative of the downward vertical force.

curacy itself. However, with reasonable support, it may be expected that a value with an uncertainty in the neighborhood of a few tenths ppm is possible within a few years.

The new ampere measurement will not only help resolve the discrepancies which now exist among several fundamental constant determinations, but will also enable the present value of $2 e / h$ used for volt monitoring applications to be brought closer to its absolute value in terms of mass, length, and time. In the future, the sensitivity of the apparatus can be used to monitor the stability of the kilogram with respect to time, by utilizing the stability of the Josephson voltage standard and the calculable capacitor. Since the force developed is sufficient to counterbalance one kilogram with only $2 m A$ in the suspended coil, the apparatus can be used to calibrate other mass standards in terms of the kilogram by merely determining a current ratio.

Even though the absolute ampere experiment as outlined above is an ambitious project, one must consider that the authors propose to measure the ampere to an accuracy 50 times better than it has ever been measured, and that absolute ampere determinations have a 100 year history of effort. Advances do not come easily.
The authors wish to thank Mr. Robert E. Kleimann and Mrs. Sheila A. Taylor for their assistance in writing the computer programs and in the operation of the computer.

\section{Appendix A}

The derivations leading to equation 8 can also be described in an integral form. This integral approach is very useful because the quantities that come into the final result are more easily measured in the laboratory.

The force in the $z$ direction between two coils of a mutual inductor is given by

$$
F_{z}=I_{1} I_{2}\left(\partial M_{12} / \partial z\right)
$$

The integral of this force from an initial position $z_{i}$ to a final position $z_{f}$ is:

$$
\int_{z_{i}}^{z_{f}} F_{z} d z=I_{1} I_{2}\left[M_{12}\left(z_{f}\right)-M_{12}\left(z_{i}\right)\right] .
$$

The emf generated in coil 1 when a current $I_{2}$ is in coil 2 is

$$
\varepsilon_{1}(t)=-I_{2}\left(\mathrm{~d} M_{12} / \mathrm{d} t\right)
$$

which can be integrated from time $t_{1}$ to $t_{2}$ :

$$
\int_{t_{1}}^{t_{2}} \varepsilon(t) d t=-I_{2}\left[M_{12}\left(t_{2}\right)-M_{12}\left(t_{1}\right)\right]
$$

Now if $t_{1}$ is the time the moving coil passes the position $z_{i}$ and $t_{2}$ the time the coil pass $z_{f}$ then we can divide eq (A2) by eq (A4)

$$
I_{1}=\left(\int_{z_{i}}^{z_{f}} F_{z} d z / \int_{t_{1}}^{t_{2}} \varepsilon_{1}(t) d t\right)
$$

This is the integral form of eq (3) and can be written explicitly in terms of NBS units so that eq (8) becomes

$K_{A} \equiv\left(A_{N B S} / A\right)=\left[\frac{\int_{z_{i}}^{z_{f}} m(z) g d z}{I_{N B S}\left(\Omega_{N B S} / \Omega\right) \int_{t_{i}}^{t_{f}} \varepsilon_{N B S}(t) d t}\right]^{1 / 2}$

The $\int_{z_{i}}^{z_{f}} m(z) g d z$ is computed from many static weighings made along the path from $z_{i}$ to $z_{f}$ with a current $I_{N B S}$ in the balance coil. The $\int_{t_{1}}^{t_{2}^{2}} \varepsilon_{N B S}(t) d t$ is measured in a separate experiment when the coil is moved from position $z_{i}$ to $z_{f}$ in a time $t_{1}-t_{2}$ at a nearly constant velocity and the current $I_{N B S}$ is not flowing in coil 1 . The geometry and the current of coil 2 must remain unchanged during all these measurement or an appropriate correction must be made. 


\section{Appendix B}

An interesting variation on this method for measuring the ampere occurs if one restricts the moveable coil to a single, rotational degree of freedom. Such a rotatable coil placed in the nearly uniform field produced by an external circuit forms the basis of the classic electrodynamometer or Pellat balance [3]

The torque on the rotatable coil is given by

$$
T(\theta)=I_{1} I_{2}\left(d M_{12} / d \theta\right)
$$

where $I_{1}$ and $I_{2}$ are the currents in the external and rotatable circuits respectively, $M_{12}$ is the mutual inductance and $\theta$ is the rotation angle. The integral of this torque in moving the coil betwen two angles is then

$W=\int_{\theta_{i}}^{\theta_{f}} T(\theta) d \theta=I_{1} I_{2} \int_{\theta_{i}}^{\theta_{f}} d M_{12}=I_{1} I_{2}\left[M_{12}\left(\theta_{f}\right)-M_{12}\left(\theta_{i}\right)\right]$

If the coil is now allowed to rotate with its circuit open, the magnitude of the emf generated is

$$
\varepsilon(t)=I_{1}\left(d M_{12} / d t\right)
$$

Integrating this emf over time one obtains

$$
\int_{t_{1}}^{t_{2}} \varepsilon(t) d t=I_{1} \int_{t_{i}}^{t_{f}} d M_{12}=I_{1}\left[M_{12}\left(t_{2}\right)-M_{12}\left(t_{1}\right)\right]
$$

If $t_{1}$ and $t_{2}$ are the times when the coil is at angles $\theta_{i}$ and $\theta_{f}$ of eq (B2) we may divide eq(B2) we may divide eq (B2) by eq (B4) to obtain

$$
I_{1}=\int_{\theta_{i}}^{\theta_{f}} T(\theta) d \theta / \int_{t_{1}}^{t_{2}} \varepsilon(t) d t
$$

Since the numerator of eq (B5) is just the work done by the electrical torque in rotating the coil between $\theta_{i}$ and $\theta_{f}$, it can be replaced with any equivalent expression for this work. In particular, this work is equal to the work done by a vertical external force applied at the end of a balance beam attached to the moveable coil when that external force just balances the electrical force. This force is what can be measured experimentally by weighing. Expressed this way eq (B5) becomes

$$
I_{1}=\int_{z_{i}}^{z_{f}} F_{z}(z) d z / \int_{t_{1}}^{t_{1}^{2}} \varepsilon(t) d t,
$$

where $z_{i}$ and $z_{f}$ are the vertical positions of the end of the balance arm at angles $\theta_{i}$ and $\theta_{f}$. This is identical to eq (A5) and leads directly to eq (A6) for $K_{A}$.

A major disadvantage of the method described in this appendix is that the force and the emf depend directly on the area of the moveable coil, in contrast to the geometry described in the body of the text, where they are essentially independent of the area of the moveable coil. Because of this, temperature control problems are more critical for the rotating coil. On the other hand, the rotary coil has the advantage of being constrained to move with one degree of freedom. As a result problems of non-vertical forces and torques which must be considered in the coil system described in the text do not affect the rotating coil. Since no displacements other than rotation are allowed, no emf's are generated and no work is done other than by the forces explicity considered.

\section{Appendix C}

The true current balance field coils will have windings of finite cross section. Below, results and specifications have been tabulated for field coils which must provide enough radial magnetic field to exert a vertical force of $4.9 \mathrm{~N}$ on a circular filament of $78 \mathrm{~cm}$ diameter containing a 10 ampere current.

The outer field coil consists of a bundle of square cross section containing $54 \times 54$ of $0.0508 \mathrm{~cm}$ diameter conductors. The mean diameter of the coil is $100 \mathrm{~cm}$.

The inner field coil consists of a bundle of rectangular

\begin{tabular}{|c|c|c|c|c|c|c|}
\hline & $z_{2}(\mathrm{~cm})$ & $z_{1}(\mathrm{~cm})$ & $\mathrm{I}_{2} \mathrm{~A}$ & $I_{1} / I_{2}$ & $\Delta F_{0}(\mathrm{ppm})$ & $2 \Delta z_{0}(\mathrm{~cm})$ \\
\hline \multirow[t]{8}{*}{$=$} & 86.1186 & 50.6786 & 59.1834593 & 1.0021216 & 7.1 & 3.5 \\
\hline & 86.2328 & 50.7328 & 59.2713175 & 1.0019726 & 12.3 & 3.9 \\
\hline & 86.3470 & 50.7848 & 59.3612670 & 1.0017318 & 19.2 & 4.5 \\
\hline & 86.5090 & 50.8712 & 59.4762047 & 1.0019700 & 33.2 & 5.1 \\
\hline & 86.5710 & 50.9024 & 59.5219867 & 1.0019812 & 39.2 & 5.4 \\
\hline & 86.6292 & 50.9314 & 59.5652223 & 1.0019806 & 45.4 & 5.6 \\
\hline & 86.7336 & 50.9832 & 59.6429316 & 1.0019745 & 58.0 & 6.2 \\
\hline & 86.8282 & 51.0302 & 59.7132118 & 1.0019770 & 70 & 6.3 \\
\hline
\end{tabular}
cross section containing 115 (radial) $\times 116$ (axial) of 0.0508 $\mathrm{cm}$ diameter conductors. The mean diameter of the coil is $24 \mathrm{~cm}$.

TABLE Cl. 


\section{Appendix D}

The coincidence of the mid-planes of each of the two coil pairs is the most critical alignment. A misalignment, in the axial direction, of the mid planes by as much as $0.004 \mathrm{~cm}$ begins to introduce an undesirable linear gradient in the force along the $z$-direction. This misalignment could be correctable by mechanically adjusting either coil pair along the axis. However figure D-l shows the positions of correction coils which provides the ability to effectively "reposition" the large field coils by + or $-0.2 \mathrm{~cm}$ in the axial or z-direction electrically. Table D-1 gives the ampere-turns, as a percent of the ampere-turns of the main coil, required to effectively reposition one of the large diameter field coils in the axial direction. Each correction coil has the same number of turns. Electrically they are connected in opposition.

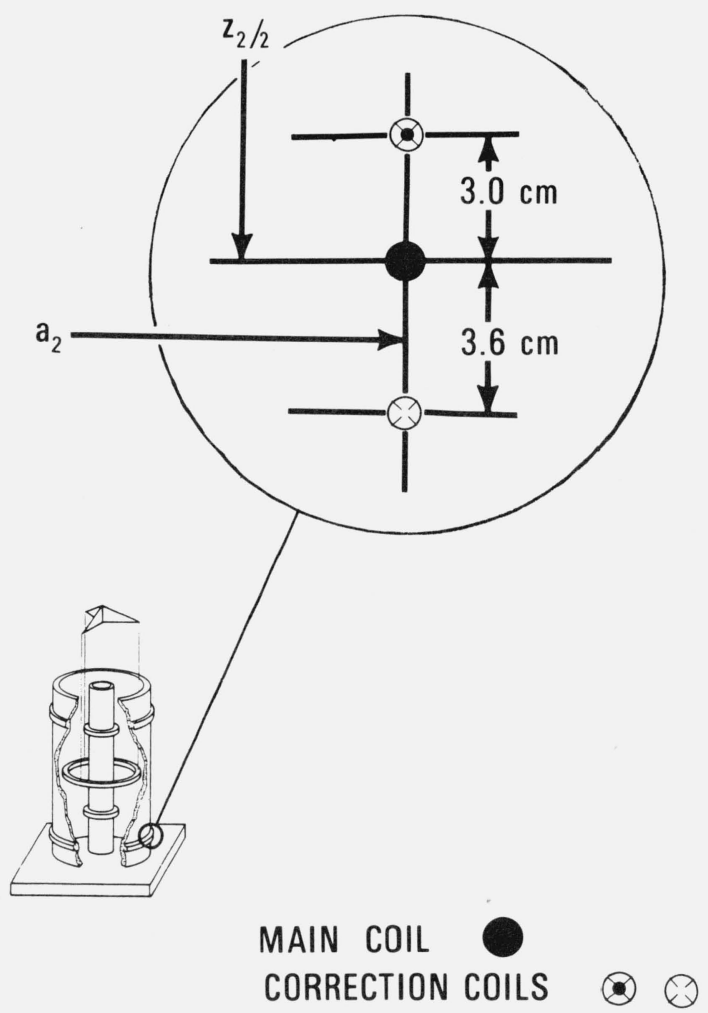

FiguRE D-1.
TABLE D-1

correction coil ampere-turns in percent of

Coil misalignment in $\mathrm{cm}$ main ampere-turns

$\begin{array}{ll}-0.20 & -3.04519 \\ -0.15 & -2.28639 \\ -0.10 & -1.52593 \\ -0.05 & -0.76380 \\ +0.05 & +0.76547 \\ +0.10 & +1.53262 \\ +0.15 & +2.30145 \\ +0.20 & +3.07197\end{array}$

\section{References}

[1] Driscoll, R. L., and Cutkosky, R. D. Measurement of Current with the National Bureau of Standards Current Balance, J. Res. Nat. Bur. Stand. (U.S.), Vol. 60, No. 4, 297-305 (April 1958).

[2] Curtis, H. L., Curtis, R. W., and Critchfield, C. L. An Absolute Determination of the Ampere, Using Improved Coils, J. Res. Nat. Bur. Stand. (U.S.), Vol. 22, 485-517 (May 1939).

[3] Driscoll, R. L. Measurement of Current with a Pellat-Type Electrodynamometer, J. Res. Nat. Bur. Stand. (U.S.), Vol. 60, No. 4, $287-$ 296 (April 1958).

[4] Driscoll, R. L., and Olsen, P. T. report to Comite Consultatif d'Electricite, Comite International des Poids at Measures, 12th Session, (1968).

[5] Taylor, B. N. Is the Present Realization of the Absolute Ampere in Error, Metrologia 12, 81 (1976).

[6] Kibble, B. P., and Robinson, I. Feasibility Study for a Moving Coil Apparatus to Relate the Electrical and Mechanical SI Units, NPL Report DES No. 40, (1977).

[7] Kibble, B. P., A Measurement of the Gyromagnetic Ratio of the Proton by the Strong Field Method, Atomic Masses and Fundamental Constants 5, ed. by J. H. Sanders and A. H. Wapstra (Plenum Publishing Corp., New York, 1976) p. 545.

[8] Cutkosky, R. D., New NBS Measurements of the Absolute Farad and Ohm, IEEE Trans. Instrum. Meas. IM-23, 305, (1974).

[9] Snow, C., Formulas for Computing Capacitance and Inductance, NBS Circular 544 (1954).

[10] Williams, E. R., and Olsen, P. T., A Non-contacting Magentic Pick-up Probe for Measuring the Pitch of a Precision Solenoid, IEEE Trans. Instrum. Meas., IM-21, 376 (1972).

[11] Olsen, P. T., and Williams, E. R., A More Accurate Determination of $\gamma_{p}$ Through Improved Dimensional Measurement Techniques, IEEE Trans. Instrum. Meas., IM-23, 302 (1974).

[12] Nakamura, H., A Serv-Controlled Balance for the Absolute Ampere Determination, Jap. J. Appl. Phys. 17, 1397, (1978). 